\title{
Probabilistic strategy-proof rules over single-peaked domains
}

Citation for published version (APA):

Peters, H. J. M., Roy, S., Sen, A., \& Storcken, A. J. A. (2013). Probabilistic strategy-proof rules over single-peaked domains. Maastricht University, Graduate School of Business and Economics. GSBE Research Memoranda No. 040 https://doi.org/10.26481/umagsb.2013040

Document status and date:

Published: 01/01/2013

DOI:

10.26481/umagsb.2013040

Document Version:

Publisher's PDF, also known as Version of record

\section{Please check the document version of this publication:}

- A submitted manuscript is the version of the article upon submission and before peer-review. There can be important differences between the submitted version and the official published version of record.

People interested in the research are advised to contact the author for the final version of the publication, or visit the DOI to the publisher's website.

- The final author version and the galley proof are versions of the publication after peer review.

- The final published version features the final layout of the paper including the volume, issue and page numbers.

Link to publication

\footnotetext{
General rights rights.

- You may freely distribute the URL identifying the publication in the public portal. please follow below link for the End User Agreement:

www.umlib.nl/taverne-license

Take down policy

If you believe that this document breaches copyright please contact us at:

repository@maastrichtuniversity.nl

providing details and we will investigate your claim.
}

Copyright and moral rights for the publications made accessible in the public portal are retained by the authors and/or other copyright owners and it is a condition of accessing publications that users recognise and abide by the legal requirements associated with these

- Users may download and print one copy of any publication from the public portal for the purpose of private study or research.

- You may not further distribute the material or use it for any profit-making activity or commercial gain

If the publication is distributed under the terms of Article $25 \mathrm{fa}$ of the Dutch Copyright Act, indicated by the "Taverne" license above, 


\section{Maastricht University}

Hans Peters, Souvik Roy,

Arunava Sen, Ton Storcken

Probabilistic Strategy-Proof Rules over Single-Peaked Domains

$\mathrm{RM} / 13 / 040$

\section{GSBE}

Maastricht University School of Business and Economics

Graduate School of Business and Economics

P.O Box 616

NL- 6200 MD Maastricht

The Netherlands 


\title{
Probabilistic Strategy-Proof Rules over Single-PeAKed Domains*
}

\author{
Hans Peters ${ }^{\dagger} \quad$ Souvik Roy ${ }^{\ddagger} \quad$ Arunava Sen $^{\S} \quad$ Ton Storcken ${ }^{\natural}$
}

July 7, 2013

\begin{abstract}
It is proved that every strategy-proof, peaks-only or unanimous, probabilistic rule defined over a minimally rich domain of single-peaked preferences is a probability mixture of strategy-proof, peaks-only or unanimous, deterministic rules over the same domain. The proof employs Farkas' Lemma and the max-flow min-cut theorem for capacitated networks.
\end{abstract}

\section{Introduction}

A probabilistic rule maps profiles of preference orderings to probability distributions over alternatives. Following the landmark paper of Gibbard (1977), a probabilistic rule is strategy-proof if the probability distribution under truth-telling stochastically dominates the probability distribution under any preference misrepresentation by any agent, for all conceivable preferences of the other agents. It is well-known that the set of strategy-proof probabilistic rules is larger than the set of strategy-proof deterministic rules; in particular all probability mixtures of strategy-proof deterministic are strategy-proof probabilistic rules. Gibbard (1977) (see also Duggan (1996) and Sen (2011)) provides a characterization of all strategy-proof probabilistic rules over the complete domain of anti-symmetric orderings. If the probabilistic rules are assumed to satisfy additional properties such as unanimity or peaks-onlyness, then all strategy-proof probabilistic rules are probability mixtures over the deterministic counterparts. A possible interpretation of this result is that randomization does not "significantly" increase the scope of mechanism design relative to the deterministic model, at least for the complete domain.

A natural question is whether the probability mixture property extends to other preference domains. Progress has been made recently for certain domains, for instance, the binary domain (Picot and Sen (2012)), the multi-dimensional domain with lexicographic preferences (Chatterji, Roy and Sen (2012) and dictatorial domains (Chatterji, Sen and Zeng (2013)). The issue appears to be subtle - it has been shown that the property holds for the first two domains but not for third, i.e., a domain on which a strategy-proof, unanimous deterministic rule is dictatorial is, in general, not one on which a strategy-proof, unanimous probabilistic rule is a random dictatorship.

The present paper establishes the probability mixture property for single-peaked domains. Specifically, every strategy-proof, peaks-only or unanimous, probabilistic rule defined on a "minimally rich" single-peaked domain is shown to be a probability mixture of strategy-proof, peaks-only or unanimous, deterministic rules over that domain. The properties hold for the domain of all single-peaked preferences since it is minimally rich. The first step of the proof establishes the property for a sub-domain of single-peaked domains, the boundary domain where the peaks of all agents lie on the boundary of the set of alternatives. This part of the proof uses Farkas' Lemma and (a version of) the max-flow min-cut theorem for capacitated networks.

\footnotetext{
*An earlier version of the paper appeared as a chapter in the second author's Ph.d thesis from Maastricht University in 2010 (Roy (2010)).

${ }^{\dagger}$ Maastricht University, Maastricht, The Netherlands.

Email: h.peters@maastrichtuniversity.nl

${ }^{\ddagger}$ Indian Statistical Institute, New Delhi, India.

Email: souvik2004@gmail.com

§Indian Statistical Institute, New Delhi, India.

Email: asen@isid.ac.in

ๆMaastricht University, Maastricht, The Netherlands.

Email: t.storcken@maastrichtuniversity.nl
} 
The second part of the proof extends the result to minimally rich domains using ideas from Ehlers, Peters and Storcken (2002).

The decomposition result on the complete single-peaked domain has been independently shown in the recent paper Pycia and Unver (2012). The proof technique in that paper is completely different from that in the present one. It is computational and relies heavily on the characterization of strategy-proof deterministic rules on single-peaked domains. In contrast, the proof methods of our paper are less direct and do not use any characterization of deterministic rules on the domain. Consequently, these methods could be useful in domains where characterizations of strategy-proof deterministic rules are difficult.

The paper is organized as follows. The next section introduces the model and definitions. Subsequent sections prove results on boundary domains and minimally rich domains. For completeness, an appendix formulates the max-flow min-cut theorem as it is used in this paper.

\section{The model}

Let $N=\{1, \ldots, n\}, n \geq 2$, denote the set of agents, who collectively have to choose an element from a finite set $A$ of alternatives. We assume $A$ to be a finite subset of the real line; in particular $A=\left\{a_{1}, \ldots, a_{m}\right\}$ with $a_{1}<\ldots<a_{m}$. A single-peaked preference ordering of agent $i$ on $A$ is a complete, reflexive, transitive and antisymmetric binary relation $P_{i}$ on $A$ satisfying the following property: there exists $\tau\left(P_{i}\right) \in A$, called the peak of $P_{i}$, such that for all $x, y \in A$, if $x<y \leq \tau\left(P_{i}\right)$ or $x>y \geq \tau\left(P_{i}\right)$ then $y P_{i} x$. Let $\mathbb{P}$ denote the set of all single-peaked preferences on $A$. A preference profile $P=\left(P_{1}, \ldots, P_{n}\right) \in \mathbb{P}^{N}$ is an $n$-tuple of single-peaked preferences. For $i \in N$, profiles $P, P^{\prime} \in \mathbb{P}^{N}$ are $i$-variants if $P_{j}=P_{j}^{\prime}$ for all $j \in N \backslash\{i\}$. Finally, $\mathcal{L}$ denotes the set of probability distributions over $A$.

Let $\mathbb{D} \subseteq \mathbb{P}$ be an arbitrary set of single-peaked preferences. A deterministic rule is a function $f: \mathbb{D}^{N} \rightarrow A$. A probabilistic rule is a function $\varphi: \mathbb{D}^{N} \rightarrow \mathcal{L}$. Thus, $\varphi(P)$ is a probability distribution over $A$ for every profile $P$. For every $a_{j} \in A, \varphi_{j}(P)$ is the probability assigned to alternative $a_{j}$ by $\varphi(P)$, i.e., $\varphi_{j}(P) \geq 0$ and $\sum_{i \in N} \varphi_{i}(P)=1$. We identify a deterministic rule with a probabilistic rule that assigns a degenerate lottery to every profile: for a deterministic rule $f$ and alternative $a_{j} \in A, f_{j}(P)=1$ if $f(P)=a_{j}$ and $f_{j}(P)=0$ otherwise. We use the notations $f(P)=a_{j}$ and $f_{j}(P)=1$ interchangeably.

Let $\mathcal{F}=\left\{f_{r} \mid r=1, \ldots, t\right\}$ be an arbitrary set of deterministic rules $f_{r}: \mathbb{D}^{N} \rightarrow A$. A probabilistic rule $\varphi: \mathbb{D}^{N} \rightarrow \mathcal{L}$ is a probability mixture of deterministic rules in $\mathcal{F}$ if there exist $\lambda_{r} \geq 0$ for $r=1, \ldots, t$ with $\sum_{r=1}^{t} \lambda_{r}=1$, such that $\varphi_{j}(P)=\sum_{r=1}^{t} \lambda_{r} f_{r j}(P)$ for all $j=1, \ldots, m$ and all $P \in \mathbb{D}^{N}$.

The probabilistic rule $\varphi$ is strategy-proof if $\sum_{k: a_{k} P_{i} a_{j}} \varphi_{k}(P) \geq \sum_{k: a_{k} P_{i}^{\prime} a_{j}} \varphi_{k}\left(P^{\prime}\right)$ for all $a_{j} \in A, i \in N$ and all $i$-variants $P, P^{\prime} \in \mathbb{D}$. Strategy-proofness requires the probability distribution from truth-telling to (weakly) stochastically dominate the probability distribution from any preference misrepresentation, for any preferences of the other agents. An equivalent interpretation of strategy-proofness is the following: the expected utility under truth-telling is (weakly) greater than the the expected utility under misrepresentation for any utility representation of the agent's true preferences and for any preferences of the other agents. Here we are following the approach of Gibbard (1977). Strategy-proofness of a deterministic rule is a special case of strategy-proofness of a probabilistic rule.

A probabilistic rule $\varphi$ satisfies unanimity if $\varphi_{j}(P)=1$ for all $P \in \mathbb{D}$ whenever $\tau\left(P_{i}\right)=a_{j}$ for all $i \in N$. This definition also extends to deterministic rules in an obvious way.

A probabilistic rule $\varphi$ satisfies peaks-onlyness if $\varphi(P)=\varphi\left(P^{\prime}\right)$ for all $P, P^{\prime} \in \mathbb{D}^{N}$ such that $\tau\left(P_{i}\right)=\tau\left(P_{i}^{\prime}\right)$ for all $i \in N$. An analogous definition holds for deterministic rules.

\section{Results on the boundary domain}

The paper provides several results on probabilistic rules on sub-domains of $\mathbb{P}$. All results follow from results on a particular sub-domain, the boundary domain $\mathbb{D}_{B}=\left\{P_{i} \mid P_{i} \in \mathbb{D}\right.$ and $\left.\tau\left(P_{i}\right) \in\left\{a_{1}, a_{m}\right\}\right\}$. The boundary domain consists of all single-peaked preferences where an agent's peak is either the left-extreme alternative $a_{1}$ or the right-extreme alternative $a_{m}$. The definition of single-peakedness implies that every preference ordering from this set is uniquely determined by the peak; in fact, the domain consists of exactly two orderings: $a_{1} P_{i} a_{2} \ldots P_{i} a_{m}$ and its reverse $a_{m} P_{i} a_{m-1} \ldots P_{i} a_{1}$. Therefore all probabilistic and deterministic rules over this domain satisfy peaks-onliness trivially.

The next result characterizes strategy-proof probabilistic rules as probability mixtures of deterministic strategy-proof rules. 
THEOREM 1. Every strategy-proof probabilistic rule $\varphi: \mathbb{D}_{B}^{N} \rightarrow \mathcal{L}$ is a probability mixture of strategy-proof deterministic rules $f: \mathbb{D}_{B}^{N} \rightarrow A$.

Proof For every $S \subseteq N, P^{S}$ will denote the boundary profile where all agents in the set $S$ have peaks at $a_{1}$ and agents in $N \backslash S$ have peaks at $a_{m}$. Let $\varphi$ be a strategy-proof probabilistic rule defined on the boundary domain. An immediate consequence of strategy-proofness is that $\sum_{i: i \leq j} \varphi_{i}\left(P^{S}\right) \leq \sum_{i: i \leq j} \varphi_{i}\left(P^{T}\right)$ for all $S \subseteq T \subseteq N$ and all $j=1, \ldots, m$.

Let $\mathcal{F}=\left\{f_{r} \mid r=1, \ldots p\right\}$ be the set of strategy-proof, deterministic rules $f_{r}: \mathcal{D}_{B}^{N} \rightarrow A$. Suppose $\varphi$ can be expressed as a linear combination over $\mathcal{F}$ with weights $\lambda_{1}, \ldots \lambda_{p}$. Then for any profile $P^{S}$

$$
1=\sum_{j=1}^{m} \varphi_{j}\left(P^{S}\right)=\sum_{j=1}^{m} \sum_{r=1}^{p} \lambda_{r} f_{r j}\left(P^{S}\right)=\sum_{r=1}^{p} \lambda_{r} \sum_{j=1}^{m} f_{r j}\left(P^{S}\right)=\sum_{r=1}^{p} \lambda_{r}
$$

so that $\sum_{r=1}^{p} \lambda_{r}=1$ holds. Therefore, in order to show that $\varphi$ is a probability mixture over $\mathcal{F}$, it suffices to show that $\lambda_{r} \geq 0$ for all $r=1, \ldots, p$. The system of equations $\varphi_{j}\left(P^{S}\right)=\sum_{r=1}^{p} \lambda_{r} f_{r j}\left(P^{S}\right)$ for all $S \subseteq N$ and $j=1, \ldots, m$ can be written in matrix form as $Z \lambda=d$ where

- $Z$ is an $2^{n} m \times p, 0$-1 matrix with rows indexed by pairs $(S, j), S \subseteq N, j \in\{1, \ldots, m\}$ and columns $r=1, \ldots, p$ such that the entry in row $(S, j)$ and column $r$ is $f_{r j}\left(P^{\bar{S}}\right)$

- $\lambda$ is a column vector of length $p$ with $\lambda_{r}$ in row $r$ and

- $d$ is a column vector of length $2^{n} m$ with $\varphi_{j}\left(P^{S}\right)$ in the row corresponding to $(S, j)$.

The proof of the theorem consists in showing that the system $Z \lambda=d$ has a nonnegative solution. By Farkas' Lemma, it is sufficient to show that $d^{\prime} y \geq 0$ for any $y \in \mathbb{R}^{2^{n} m}$ with $Z^{\prime} y \geq \mathbf{0} \in \mathbb{R}^{p}$. Denoting the component of the vector $y$ corresponding to $(S, j)$ by $y(S, j)$, the latter system of inequalities can be written as

$$
\sum_{S \subseteq N} \sum_{j=1}^{m} f_{r j}\left(P^{S}\right) y(S, j) \geq 0 \text { for all } r=1, \ldots, p .
$$

For all $r \in\{1, \ldots, p\}$ and $S \subseteq N$ let $r(S) \in\{1, \ldots, m\}$ be such that $f_{r}\left(P^{S}\right)=a_{r(S)}$. Then $f_{r}$ is strategyproof if and only if $r(S) \geq r(T)$ whenever $S \subseteq T$. Thus, (1) and strategy-proofness of $f_{r}, r=1, \ldots, p$, are equivalent to the following:

$$
\sum_{S \subseteq N} y(S, r(S)) \geq 0 \text { for all }(S, r(S))_{S \subseteq N} \text { with } r(S) \geq r(T) \text { whenever } S \subseteq T .
$$

We thus have to prove that if $y \in \mathbb{R}^{2^{n} m}$ satisfies (2) then

$$
\sum_{S \subseteq N} \sum_{j=1}^{m} y(S, j) \varphi_{j}\left(P^{S}\right) \geq 0
$$

This will be proved using a network-flow formulation of the problem (see Appendix). The network is defined as follows. Consider an arbitrary numbering of the sets $S \subseteq N, S_{1}, S_{2}, \ldots, S_{2^{n}}$ with $S_{1}=\emptyset$ and $S_{2^{n}}=N$. The set of vertices is

$$
V=\{\alpha, \beta\} \cup\left\{\left(S_{i}, j\right) \mid i=1, \ldots, 2^{n}, j=1, \ldots, m\right\} .
$$

Here, $\alpha$ is the source and $\beta$ is the sink. All other vertices are described by a subset of agents and an alternative. The edges in the graph are determined by the set of deterministic rules, in the following way.

- For every $r \in\{1, \ldots, p\}$ let $E_{r}=\left\{\left(\left(S_{i}, j\right),\left(S_{i+1}, k\right)\right) \mid i=1, \ldots 2^{n}-1, f_{r j}\left(P^{S_{i}}\right)=f_{r k}\left(P^{S_{i+1}}\right)=1\right\}$.

- There is an edge $\left(\alpha,\left(S_{1}, j\right)\right)$ for each $j=1, \ldots, m$.

- There is an edge $\left(\left(S_{2^{n}}, j\right), \beta\right)$ for each $j=1, \ldots, m$.

Now the set of edges $E$ is the union of the sets $E_{r}$, the set of $m$ edges starting at $\alpha$, and the set of $m$ edges ending at $\beta$. Observe that every deterministic, strategy-proof rule generates a path from the source $\alpha$ to the $\operatorname{sink} \beta$.

The capacities of the vertices of the network are defined as follows. 
- The capacity of the vertex $\left(S_{i}, j\right), i=1, \ldots, 2^{n}, j=1, \ldots, m$ is $c\left(S_{i}, j\right)=\varphi_{j}\left(P^{S_{i}}\right)$.

- $c(\alpha)=c(\beta)=1$.

Probabilities are thought of as the flow through the network. The paths in the network are determined by deterministic, strategy-proof rules. The goal of the argument is to show that the maximal flow exhausts the capacity at every vertex. This is done by using a version of the max-flow min-cut theorem.

Claim 1 The minimum capacity of a cut is equal to 1.

The proof of Claim 1 proceeds in three steps. Let $C$ be a cut not containing $\alpha$ or $\beta$.

Step 1 There exist $S_{i_{1}}, \ldots, S_{i_{m}}$ such that $S_{i_{j+1}} \subseteq S_{i_{j}}$ for all $j=1, \ldots, m-1$ and $\left(S_{i_{j}}, j\right) \in C$ for all $j=1, \ldots, m$.

To prove this, observe first that there must exist some $\left(S_{i_{k}}, k\right) \in C$ for all $k \in\{1, \ldots, m\}$. To see this, let $k \in\{1, \ldots, m\}$ and consider the path $\left(\alpha,\left(S_{1}, k\right),\left(S_{2}, k\right), \ldots,\left(S_{2^{n}}, k\right), \beta\right)$ generated by the constant (strategyproof) deterministic rule $f_{r k}\left(P^{S_{i}}\right)=1$ for all $S^{i} \subseteq N$. Since $C$ is a cut, this path must intersect $C$. Hence, the cut $C$ can be written in the form

$$
C=\left\{\left(S_{1,1}, 1\right), \ldots,\left(S_{1, k_{1}}, 1\right),\left(S_{2,1}, 2\right), \ldots,\left(S_{2, k_{2}}, 2\right), \ldots,\left(S_{m, 1}, m\right), \ldots,\left(S_{m, k_{m}}, m\right)\right\} .
$$

We define a deterministic rule $f$ by the following algorithm. Let $S \subseteq N$.

- If there are $\left(S_{i_{m-1}}, m-1\right), \ldots,\left(S_{i_{1}}, 1\right) \in C$ such that $S \subseteq S_{i_{m-1}} \subseteq \ldots \subseteq S_{i_{1}}$, then $f\left(P^{S}\right)=a_{m}$.

- Else, if there are $\left(S_{i_{m-2}}, m-2\right), \ldots,\left(S_{i_{1}}, 1\right) \in C$ such that $S \subseteq S_{i_{m-2}} \subseteq \ldots \subseteq S_{i_{1}}$, then $f\left(P^{S}\right)=a_{m-1}$.

- ...

- Else, if there are $\left(S_{i_{k-1}}, k-1\right), \ldots,\left(S_{i_{1}}, 1\right) \in C$ such that $S \subseteq S_{i_{k-1}} \subseteq \ldots \subseteq S_{i_{1}}$, then $f\left(P^{S}\right)=a_{k}$.

- ...

- Else, if there are $\left(S_{i_{2}}, 2\right),\left(S_{i_{1}}, 1\right) \in C$ such that $S \subseteq S_{i_{2}} \subseteq S_{i_{1}}$, then $f\left(P^{S}\right)=a_{3}$.

- Else, if there is $\left(S_{i_{1}}, 1\right) \in C$ such that $S \subseteq S_{i_{1}}$, then $f\left(P^{S}\right)=a_{2}$.

- In all remaining cases, $f\left(P^{S}\right)=a_{1}$.

It is not difficult to verify that this rule $f$ is strategy-proof, i.e., $f=f_{r}$ for some $r \in\{1, \ldots, p\}$. Since $C$ is a cut, the path generated by $f_{r}$ must intersect $C$, say in $(S, k)$, so that in particular $f\left(P^{S}\right)=a_{k}$. Suppose $k<m$. Then by definition of $f_{r}=f$ there are $\left(S_{i_{k-1}}, k-1\right), \ldots,\left(S_{i_{1}}, 1\right) \in C$ such that $S \subseteq S_{i_{k-1}} \subseteq \ldots \subseteq S_{i_{1}}$. Since $(S, k) \in C$, we obtain $S_{i_{k}}=S \subseteq S_{i_{k-1}} \subseteq \ldots \subseteq S_{i_{1}}$ for some $\left(S_{i_{k}}, k\right) \in C$, but then $f\left(P^{S}\right) \neq a_{k}$ by definition of $f_{r}$, a contradiction. Hence $k=m$, which implies that there are $\left(S_{i_{m}}, m\right), \ldots,\left(S_{i_{1}}, 1\right) \in C$ such that $S_{i_{m}}=S \subseteq S_{i_{m-1}} \subseteq \ldots \subseteq S_{i_{1}}$. This completes the proof of Step 1 .

Step 2 The capacity of $C$ is at least 1.

To prove this, according to Step 1 we can take $S_{i_{1}}, \ldots, S_{i_{m}}$ such that $S_{i_{j+1}} \subseteq S_{i_{j}}$ for all $j=1, \ldots, m-1$ and $\left(S_{i_{j}}, j\right) \in C$ for all $j=1, \ldots, m$. Then

$$
c\left(S_{i_{1}}, 1\right)+c\left(S_{i_{2}}, 2\right)=\varphi_{1}\left(P^{S_{i_{1}}}\right)+\varphi_{2}\left(P^{S_{i_{2}}}\right) \geq \varphi_{1}\left(P^{S_{i_{2}}}\right)+\varphi_{2}\left(P^{S_{i_{2}}}\right),
$$

where the inequality follows from strategy-proofness of $\varphi$ and the fact that $S_{i_{1}} \supseteq S_{i_{2}}$. Suppose

$$
c\left(S_{i_{1}}, 1\right)+c\left(S_{i_{2}}, 2\right)+\ldots+c\left(S_{i_{k}}, k\right) \geq \varphi_{1}\left(P^{S_{i_{k}}}\right)+\ldots+\varphi_{k}\left(P^{S_{i_{k}}}\right)
$$

for some $k \in\{2, \ldots, m-1\}$. Then

$$
\begin{aligned}
c\left(S_{i_{1}}, 1\right)+\ldots+c\left(S_{i_{k}}, k\right)+c\left(S_{i_{k+1}}, k+1\right) & \geq \varphi_{1}\left(P^{S_{i_{k}}}\right)+\ldots+\varphi_{k}\left(P^{S_{i_{k}}}\right)+\varphi_{k+1}\left(P^{S_{i_{k+1}}}\right) \\
& \geq \varphi_{1}\left(P^{S_{i_{k+1}}}\right)+\ldots+\varphi_{k}\left(P^{S_{i_{k+1}}}\right)+\varphi_{k+1}\left(P^{S_{i_{k+1}}}\right) .
\end{aligned}
$$

The second inequality follows again from strategy-proofness of $\varphi$ and the fact that $S_{i_{k}} \supseteq S_{i_{k+1}}$. It follows from induction that

$$
c(C) \geq c\left(S_{i_{1}}, 1\right)+\ldots+c\left(S_{i_{m}}, m\right) \geq \varphi_{1}\left(P^{S_{i_{m}}}\right)+\ldots+\varphi_{m}\left(P^{S_{i_{m}}}\right)=1 .
$$


This completes the proof of Step 2.

Step 3 The minimum capacity of a cut is equal to 1.

To prove this, note that the capacity of any cut containing $\alpha$ or $\beta$ is 1 . Hence, by Step 2 , the minimum capacity of a cut is 1 .

The proof of Claim 1 is now complete.

By Claim 1 and the max-flow min-cut theorem, the maximal flow through the network has value 1. Suppose that at some flow through the network, the flow through some vertex $\left(S_{i}, j\right)$ is less than its capacity $\varphi_{j}\left(P^{S_{i}}\right)$. Consider the cut $\left\{\left(S_{i}, 1\right), \ldots,\left(S_{i}, m\right)\right\}$. The flow through this cut is then strictly less than $\sum_{k=1}^{m} \varphi_{k}\left(P^{S_{i}}\right)=1$. Hence, the flow is not maximal. Thus, a maximal flow through the network exhausts the capacity of every vertex.

The proof of the theorem can now be completed. Consider a maximal flow through the network. From the definition of the network, a path is determined by a deterministic, strategy-proof rule. For any such rule $f_{r}$ let $F l(r)$ denote the flow through this path. Clearly, $F l(r) \geq 0$. Hence by (2) we have

$$
\sum_{r=1}^{p} \sum_{S \subseteq N} y(S, r(S)) F l(r) \geq 0
$$

Consider the coefficient of an arbitrary term $y(S, j)$ at the left-hand side of (5). The total flow at the vertex $(S, j)$ in the network is the sum of the flows through the vertex through all the paths. It has just been established that the flow through this vertex must be the capacity of the vertex, i.e. $\varphi_{j}\left(P^{S}\right)$. Hence

$$
\sum_{S \subseteq N} \sum_{j=1}^{m} y(S, j) \varphi_{j}\left(P^{S}\right)=\sum_{r=1}^{p} \sum_{S \subseteq N} y(S, r(S)) F l(r) \geq 0
$$

so that (3) holds, as required.

The next result shows that Theorem 1 can be extended to unanimous probabilistic and deterministic rules.

THEOREM 2. Every strategy-proof, unanimous probabilistic rule $\varphi: \mathbb{D}_{B}^{N} \rightarrow \mathcal{L}$ is a probability mixture of strategy-proof, unanimous deterministic rules $f: \mathbb{D}_{B}^{N} \rightarrow A$.

Proof Only minor modifications of the proof of Theorem 1 are required - specifically only of the proof of Claim 1. Unanimity implies $\varphi_{m}\left(P^{\emptyset}\right)=\varphi_{1}\left(P^{N}\right)=1$. Recall that in the proof of Claim $1, S_{1}=\emptyset$ and $S_{2^{n}}=N$, i.e. $c\left(S_{1}, m\right)=c\left(S_{2^{n}}, 1\right)=1$ and $c\left(S_{1}, j\right)=c\left(S_{2^{n}}, i\right)=0$ for all $j \neq m$ and $i \neq 1$. Any cut that contains either $\left(S_{1}, m\right)$ or $\left(S_{2^{n}}, 1\right)$ must have capacity one. Moreover, any vertices in the cut of the form $\left(S_{1}, j\right)$ and $\left(S_{2^{n}}, i\right), j \neq m, i \neq 1$ can be deleted from the cut without changing its capacity. Consider a reduced network by deleting vertices of the form $\left(S_{1}, j\right)$ and $\left(S_{2^{n}}, i\right), j \neq m, i \neq 1$. All the arguments in the proof of Claim 1 go through for the reduced network, i.e the minimum capacity of a cut is one. No other modifications of the proof of Theorem 1 are required.

Theorems 1 and 2 result in the following corollary for the binary domain by observing that, if $A=\left\{a_{1}, a_{2}\right\}$, then the complete domain consists of the two orderings $a_{1} P_{i} a_{2}$ and $a_{2} P_{i} a_{1}$. Hence, in that case the complete domain coincides with the boundary domain.

Corollary 1. Suppose $|A|=2$.

(a) Every strategy-proof probabilistic rule defined on the complete domain is a probability mixture of strategy-proof deterministic rules.

(b) Every strategy-proof, unanimous probabilistic rule defined on the complete domain is a probability mixture of strategy-proof, unanimous deterministic rules.

Corollary 1(a) was earlier proved in Picot and Sen (2012). 


\section{Extensions to minimally rich sub-domains of single-peaked pref- erences}

The goal of this section is to provide extensions of the results on boundary domains to supersets of this domain.

A single-peaked ordering $P_{i} \in \mathbb{P}$ is left-extreme if $a_{j}<\tau\left(P_{i}\right)<a_{k}$ implies $a_{j} P_{i} a_{k}$. Similarly, a single-peaked ordering $P_{i} \in \mathbb{P}$ is right-extreme if $a_{j}<\tau\left(P_{i}\right)<a_{k}$ implies $a_{k} P_{i} a_{j}$. A domain $\mathbb{D} \subseteq \mathbb{P}$ is minimally rich if it contains all left and right-extreme single-peaked orderings. In other words, every alternative $a_{j}$ is the peak of at least two orderings $P_{i}, P_{i}^{\prime} \in \mathbb{D}$ where $a_{j} P_{i} a_{j-1} \ldots a_{1} P_{i} a_{j+1} \ldots P_{i} a_{m}$ and $a_{j} P_{i}^{\prime} a_{j+1} \ldots a_{m} P_{i}^{\prime} a_{j-1} \ldots P_{i}^{\prime} a_{1}$. In particular, the domain $\mathbb{P}$ is minimally rich.

Theorems 1 and 2 can be extended to minimally rich domains.

TheOrem 3. Let $\mathbb{D} \subseteq \mathbb{P}$ be a minimally rich domain.

(a) Every strategy-proof, peaks-only probabilistic rule $\varphi: \mathbb{D}^{N} \rightarrow \mathcal{L}$ is a probability mixture of strategy-proof, peaks-only deterministic rules $f: \mathbb{D}^{N} \rightarrow A$.

(b) Every strategy-proof, unanimous probabilistic rule $\varphi: \mathbb{D}^{N} \rightarrow \mathcal{L}$ is a probability mixture of strategy-proof, unanimous deterministic rules $f: \mathbb{D}^{N} \rightarrow A$.

Both results in Theorem 3 derive from the fact the the outcome of a strategy-proof and peaks-only or unanimous rule at any profile is determined completely by outcomes of the same rule at boundary profiles. Details are provided below.

For any pair of alternatives $a_{j}, a_{k}, \operatorname{conv}\left(a_{j}, a_{k}\right)$ is the set of alternatives (including $a_{j}$ and $\left.a_{k}\right)$ that lie between $a_{j}$ and $a_{k}$. A probabilistic rule $\varphi: \mathbb{D}^{N} \rightarrow \mathcal{L}$ is uncompromising if $\sum_{j: a_{j} \in B} \varphi_{j}(P)=\sum_{j: a_{j} \in B} \varphi_{j}\left(P^{\prime}\right)$ for all $i \in N, i$-variants $P, P^{\prime} \in \mathbb{D}^{N}$, and $B \subseteq A$ such that $B \cap \operatorname{conv}\left(\tau(P), \tau\left(P^{\prime}\right)=\emptyset\right.$. Suppose an agent moves his peak from $a_{j}$ to $a_{k}$. If a probabilistic rule is uncompromising, the probability of all subsets of alternatives not intersecting the interval between $a_{j}$ and $a_{k}$ is unchanged. This definition was introduced in the deterministic model by Border and Jordan (1983) and extended to the probabilistic model by Ehlers, Peters and Storcken (2002).

Proposition 1. Let $\mathbb{D} \subseteq \mathcal{P}$ be an arbitrary domain with $\mathbb{D}_{B} \subseteq \mathbb{D}$. Let $\varphi^{1}: \mathbb{D}^{N} \rightarrow \mathcal{L}$ and $\varphi^{2}: \mathbb{D}^{N} \rightarrow \mathcal{L}$ be uncompromising probabilistic rules such that $\varphi^{1}(P)=\varphi^{2}(P)$ for all $P \in \mathbb{D}_{B}^{N}$. Then $\varphi^{1}(P)=\varphi^{2}(P)$ for all $P \in \mathbb{D}^{N}$.

Proof Let $a_{j}$ be an arbitrary alternative and let $P \in \mathbb{D}^{N}$. Let $S, T \subseteq N$ be such that $S=\left\{i \mid \tau\left(P_{i}\right) \leq a_{j}\right\}$ and $T=\left\{i \mid \tau\left(P_{i}\right)<a_{j}\right\}$. Since $\varphi^{1}$ and $\varphi^{2}$ are uncompromising, $\varphi_{\left\{k: a_{k} \leq a_{j}\right\}}^{1}(P)=\varphi_{\left\{k: a_{k} \leq a_{j}\right\}}^{1}\left(P^{S}\right)$ and $\varphi_{\left\{k: a_{k}<a_{j}\right\}}^{1}(P)=\varphi_{\left\{k: a_{k}<a_{j}\right\}}^{1}\left(P^{T}\right)$ and $\varphi_{\left\{k: a_{k} \leq a_{j}\right\}}^{2}(P)=\varphi_{\left\{k: a_{k} \leq a_{j}\right\}}^{2}\left(P^{S}\right)$ and $\varphi_{\left\{k: a_{k}<a_{j}\right\}}^{2}(P)=\varphi_{\left\{k: a_{k}<a_{j}\right\}}^{2}\left(P^{T}\right){ }^{1}$ Hence,

$$
\begin{aligned}
\varphi_{j}^{1}(P) & =\varphi_{\left\{k: a_{k} \leq a_{j}\right\}}^{1}(P)-\varphi_{\left\{k: a_{k}<a_{j}\right\}}^{1}(P) \\
& =\varphi_{\left\{k: a_{k} \leq a_{j}\right\}}^{1}\left(P^{S}\right)-\varphi_{\left\{k: a_{k}<a_{j}\right\}}^{1}\left(P^{T}\right) \\
& =\varphi_{\left\{k: a_{k} \leq a_{j}\right\}}^{2}\left(P^{S}\right)-\varphi_{\left\{k: a_{k}<a_{j}\right\}}^{2}\left(P^{T}\right) \\
& =\varphi_{\left\{k: a_{k} \leq a_{j}\right\}}^{2}(P)-\varphi_{\left\{k: a_{k}<a_{j}\right\}}^{2}(P) \\
& =\varphi_{j}^{2}(P)
\end{aligned}
$$

which completes the proof.

The proof of Theorem 3 can now be completed.

Proof of Theorem 3 Ehlers, Peters and Storcken (2002) show that (i) strategy-proofness and peaksonlyness imply uncompromisingness and (ii) strategy-proofness and unanimity imply uncompromisingness over $\mathbb{P}$. However, their arguments carry over for any minimally rich domain. Let $\varphi$ be a strategy-proof and peaks-only or unanimous rule over a minimally rich domain. Theorems 1 and 2 imply that there exist $\lambda_{r}, \ldots, \lambda_{p}$ such that $\varphi_{j}\left(P^{S}\right)=\sum_{r=1}^{p} \lambda_{r} f_{r j}\left(P^{S}\right)$ for all boundary profiles $P^{S} \in \mathbb{D}_{B}^{N}$. Theorem 3 now follows by applying Proposition 1 .

\footnotetext{
${ }^{1}$ Here, $\varphi_{\left\{k: a_{k} \leq a_{j}\right\}}^{1}(P)=\sum_{k: a_{k} \leq a_{j}} \varphi_{k}^{1}(P)$, etc.
} 
ObSERvation 1. The decomposition results on minimally rich domains are not valid without the peaks-only or unanimity assumptions. It is well-known that unilateral schemes in Gibbard (1977) are not probability mixtures of strategy-proof deterministic rules.

OBSERVATION 2. Theorem 3 provides full characterizations of strategy-proof and peaks-only or unanimous probabilistic rules in conjunction with the established characterizations of strategy-proof and peaks-only or unanimous deterministic rules (see Moulin (1980), Barberà, Gul and Stachetti (1993) and Weymark (2012)). These results are derived for the complete single-peaked domain $\mathbb{P}$. However, our Proposition 1 implies that they hold for any minimally rich domain. Finally, in contrast to Pycia and Unver (2012), the proof in the present paper does not rely on the characterization of deterministic rules.

\section{Appendix}

A network is a directed graph $G=(V, E)$, with $V$ the finite set of vertices and $E \subseteq V \times V$ the set of edges, and with $s, t \in V$ being the source (having only outgoing edges) and the sink (having only ingoing edges) of $G$, respectively. The capacity of a vertex $v \in V$ is a nonnegative number $c(v)$. A flow is a mapping $F: E \rightarrow \mathbb{R}_{+}$satisfying the following constraints:

(F1) $\sum_{u:(u, v) \in E} F(u, v)=\sum_{u:(v, u) \in E} F(v, u)$, for each $v \in V \backslash\{s, t\}$,

(F2) $\sum_{u \in V} F(u, v) \leq c(v)$ for each $v \in V \backslash\{s\}$.

(F3) $\sum_{v \in V} F(s, v) \leq c(s)$.

The value of a flow $F$ is defined as the amount $\sum_{v \in V} F(s, v)$. It represents the amount of flow passing from the source to the sink.

A path from $s$ to $t$ is a sequence of vertices $s=v_{1}, \ldots, v_{\ell}=t$ such that $\left(v_{i}, v_{i+1}\right) \in E$ for all $i=1, \ldots, \ell-1$. A cut $C$ is a subset of $V$ such that for any path from $s$ to $t$ there is a $u$ on this path with $u \in C$. The capacity of a cut $C$, denoted by $c(C)$, is the sum of the capacities of all its elements. Clearly, the value of any flow is smaller or equal to the capacity of any cut. The following classical result - presented here in a version with capacities on the vertices - is from Ford and Fulkerson (1956). ${ }^{2}$

Max-flow min-cut theorem: The maximum value of a flow is equal to the minimum capacity of a cut.

\section{References}

Ahuja RK, Magnanti TL, Orlin JB (1993) Network Flows. Prentice Hall Inc., New Jersey.

Barberà S, Gul F, Stachetti E (1993) Generalized Median Voter Schemes and Committees. Journal of Economic Theory 61:262-289.

Border K, Jordan J (1983) Straightforward Elections, Unanimity and Phantom Voters. Review of Economic Studies 50:153-170.

Chatterji S, Roy S, Sen A (2012) The Structure of Strategy-proof Random Social Choice Functions over Product Domains and Lexicographically Separable Domains. Journal of Mathematical Economics 48:353-366.

Chatterji S, Sen A, Zeng H (2013) Random Dictatorship Domains. Mimeo.

Duggan J (1996) A Geometric Proof of Gibbard's Random Dictatorship Theorem. Economic Theory 7:365369.

Ehlers L, Peters H, Storcken A (2002) Strategy-proof Probabilistic Decision Schemes for One-dimensional Single-Peaked Preferences. Journal of Economic Theory 105:408-434.

Gibbard A (1977) Manipulation of Voting Schemes that Mix Voting With Chance. Econometrica 45:665681.

\footnotetext{
${ }^{2}$ The so-called generalized max-flow min-cut theorem allows for capacities on both the edges and the vertices. The version formulated here is obtained from this by setting the edge capacities high. See, e.g., Ahuja, Magnanti, and Orlin (1993).
} 
Ford LR, Fulkerson DR (1956) Maximal flow through a network. Canadian Journal of Mathematics 8:399404.

Moulin H (1980) On Strategy-proofness and Single Peakedness. Public Choice 35:437-455.

Picot J, Sen A (2012) An Extreme Point Characterization of Random Strategy-proof Social Choice Functions: The Two Alternatives Case. Economic Letters 115:49-52.

Pycia, M, Unver U (2012) Decomposing Random Mechanisms. Mimeo.

Roy S (2010) Manipulability, Decomposability and Rationalizability. Ph.d Thesis, Maastricht University, The Netherlands.

Sen, A (2011) The Gibbard Random Dictatorship Theorem: A Generalization and a New Proof. Spanish Economic Review, SERIEs 2(4):515-527.

Weymark J (2011) A Unified Approach to Strategy-proofness for Single-Peaked Preferences. Spanish Economic Review, SERIEs 2(4):529-550. 